Francis MANZANO

Université Rennes-2 Haute Bretagne Laboratoire "Ethnotextes, variations et pratiques dialectales"

(C.E.L.L.A.M.)

\title{
SUR LES MÉCANISMES DU PAYSAGE SOCIOLINGUISTIQUE ET IDENTITAIRE D'AFRIQUE DU NORD. ${ }^{1}$
}

\section{Première partie : Genèse du système tripolaire}

\section{Avant-propos ${ }^{2}$}

Les soubresauts récents au Maghreb, ceux de l'Algérie en particulier, font apparaître souvent de manière violente une série de tensions identitaires et sociolinguistiques très typiques des personnalités collectives d'Afrique du Nord.

Pour comprendre ce qui se passe et ce qui pourrait se passer bientôt, il est bon de s'interroger sur la dynamique globale du système des langues en présence, à condition de se donner des moyens de contrôle en profondeur. De ce point de vue, il est probable qu'un regard trop synchronique conduit à produire des simplifications que l'on pourrait considérer comme politiquement légitimes, mais scientifiquement critiquables. L'une de ces simplifications constantes dans la littérature technique ou émotionnelle depuis une quarantaine d'années, consiste à enfermer les conflits sociolinguistiques dans un déroulement historique et politique récent: celui de la colonisation française, puis de la décolonisation. Un emboîtement binaire qui présente plusieurs défauts. Il conduit en effet à un débat réduit et piégé sur des dichotomies du type national/non-national, local/véhiculaire, imposé/choisi, authentique/non authentique, et pose ces différentes paires comme fondées par la colonisation française, en masquant la permanence historique du contact des langues en Afrique du Nord. Il conduit ensuite à un deuxième emboîtement, logé dans le précédent, celui de

\footnotetext{
${ }^{1}$ Première parution : « Langage et Société », $\mathrm{n}^{\circ} 75$, mars 1996.

${ }^{2}$ Les principales références bibliographiques sont données en bas de page, et non reprises en fin d'article.
} 
l'affrontement direct entre arabe et français, ce qui amène à évacuer ou marginaliser de facto le berbère. Autre piège, et non des moindres, celui qui enferme les locuteurs dans les cadres préalables qui précèdent. Tout acteur du paysage linguistique maghrébin (surtout durant les deux décennies 1960-1980) est presque obligé d'admettre comme légitimes les dialectiques colonisation/décolonisation, français/arabe. Ne pas le faire reviendrait à se mettre en position de déviance, position toujours délicate dans les groupements nordafricains où la pression sociale est particulièrement forte. Il s'en suit alors une forme de névrose très perceptible quand les gens parlent des langues au Maghreb. Peuvent alors se croiser propos positifs et propos négatifs : on aime et on déteste, on accueille et on rejette à quelques mots de distance, mais cela concerne toutes les langues en présence.

\section{Faiblesses d'une structuration binaire: trois pôles et non deux.}

L'organisation binaire qui vient d'être suggérée rend très mal compte des réalités historiques des langues, comme on va le voir. Elle interdit en outre toute perception dynamique du système d'interaction au sein du marché linguistique dès lors qu'elle conduit à privilégier certains couples là où les relations sont en fait plus complexes. Il est certes difficile d'échapper aux travers d'une modélisation. Du moins peut-on s'efforcer d'avoir une vue d'ensemble qui permette en même temps de rendre compte des mouvements du passé et de contrôler les principales tendances à l'oeuvre à la fin du $\mathrm{XX}^{\text {ème }}$ siècle.

\section{2-1. La question du français et du pôle roman.}

On fait généralement comme si le français était une langue importée, par une sorte d'irruption imprévisible, comme en Afrique subsaharienne ou en Asie par exemple. Il ne s'agit pas de remettre ici en question le rôle évident de la colonisation (Algérie) ou des protectorats (Maroc, Tunisie) dans l'implantation statutaire du français; mais il convient de préciser que l'influence de cette langue est en réalité antérieure à l'implantation strictement politique de la France et que l'installation du français s'inscrit dans un processus beaucoup plus profond. Tout d'abord, certaines études tendent à montrer que le français était utilisé avant l'acte politique de la colonisation ${ }^{3}$. Dans les élites et la bourgeoisie commerçante certainement, plus généralement au sein de groupes et chez des individus en contact avec la France et l'Europe.

D'autre part, réduire la francisation à la colonisation fait perdre de vue que le contact avec la Romania, avec des hauts et des bas, n'a jamais été interrompu. De ce point de vue, le Maghreb, de longue date et aujourd'hui encore, est situé sur une ligne de faille entre famille romane et famille chamito-sémitique. Cette ligne se déplace et la francisation n'est jamais qu'une concrétisation relativement récente d'un pôle roman constitutif du paysage maghrébin.

\footnotetext{
${ }^{3}$ Par exemple, études publiées ou en cours inédites de Hichem Skik sur la Tunisie (différents travaux, en particulier dans les Cahiers $d u$ CERES, Tunis).
} 
C'est ce que nous montrerons dans un premier temps.

\section{2-1-a. La Romania primitive.}

On doit rappeler que le Maghreb a constitué pour les romanistes une section importante de la "Romania perdue" ${ }^{4}$. On désignait ainsi l'ensemble des régions fortement romanisées et progressivement perdues par les langues romanes, ou gagnées par d'autres groupes de langues: langues slaves à l'est, langues germaniques au nord, langues chamito-sémitiques au sud.

Les débuts du latin en Afrique du Nord datent de la deuxième moitié du II $^{\text {ème }}$ siècle av. J.-C. La chronologie de cette latinisation ne peut être établie très précisément, et on se contentera ici de délimiter de grands cadres.

Ce fut semble-t-il une latinisation plutôt élitaire jusqu'au II ${ }^{\text {ème }}$ siècle de l'ère chrétienne. Il est intéressant de noter que durant cette période le latin bute visiblement sur le punique (ou phénicien) dans les villes et les circuits commerciaux, le berbère (ou libyque) dans les campagnes. Il avance alors par le biais d'un mouvement d'acculturation des classes hautes, lesquelles semblent rester longtemps bi- ou trilingues pour échanger avec leur peuple. Situation de pluriglossie donc, attestée bien plus tard par des témoignages comme celui d'Augustin $^{5}$.

Du II ${ }^{\text {ème }}$ au IV $^{\text {ème }}$ siècle ap. J.-C., le mouvement s'approfondit. Le latin touche désormais les classes populaires, car il est étroitement lié à la propagation du christianisme. Nous avons de cela des preuves convergentes. Se multiplient les matériaux d'évangélisation (fragments bibliques en latin populaire), les épitaphes chrétiennes révélatrices des mutations en cours au sein du latin vulgaire ${ }^{6}$, les oeuvres littéraires "africaines" dans l'inspiration comme dans la forme ${ }^{7}$. On peut donc concevoir cette période comme une phase de fixation de la Romania d'Afrique, avec dégagement probable de latins locaux ou régionaux.

Comme ailleurs dans la Romania, cette localisation était en passe de déboucher sur la production d'idiomes romans spécifiques à l'arrivée des arabes (VII ${ }^{\text {ème }}$ siècle).

La plupart du temps, on tient à ce sujet un raisonnement très caractéristique $\mathrm{du}$ réductionnisme dont nous avons parlé. Ce serait une Romania mort-née, éradiquée rapidement par l'arabisation, d'autant plus facilement que la latinisation serait toujours restée superficielle.

\footnotetext{
${ }^{4}$ Voir par exemple Willy Bal : Introduction aux études de linguistique romane, avec considération spéciale de la langue française. Paris, Didier, 1966.

${ }^{5} \mathrm{~A}$ l'époque de Saint-Augustin (fin $\mathrm{IV}^{\mathrm{eme}}$-début $\mathrm{V}^{\mathrm{e} m}$ ), et suivant le témoignage de ce dernier, semblent couramment parlés le berbère et le punique dans les milieux populaires.

${ }^{6}$ Les inscriptions funéraires chrétiennes se multiplient à partir du IIIème siècle. Elles reproduisent pour la plupart des traits phonétiques proto-romans que l'on retrouve ailleurs dans la Romania ou certains secteurs de celle-ci. A ce moment circulent aussi des adaptations locales du Nouveau Testament, Evangiles de Saint Luc et Saint Jean par exemple, dont certains écrivains latinophones d'Afrique ont transmis les fragments.

${ }^{7}$ De Tertullien à Commodien se produit une convergence dans les types de fautes (confusions de cas, tournures analytiques romanes etc.) ou dans l'inspiration même (par exemple fortes tendances mystiques ou catastrophiques) qui semble globalement singulariser les auteurs africains à cette époque.
} 
Cet argument de la superficialité est réfuté par l'abondance des preuves antérieures (épigraphie, littérature).

Il l'est également par des preuves actuelles. En effet, les systèmes macro- et microtoponymiques actuels (Algérie orientale et Tunisie surtout, à un degré moindre Algérie occidentale et Maroc) conservent la trace de mutations phonétiques proto-romanes en cours aux VIIème siècle, dans la prolongation des épitaphes des premiers siècles de l'ère. Ces traces peuvent être repérées sur de la toponymie d'origine latine et, ce qui est plus significatif encore, sur des matériaux non romans relevant le plus souvent du fond libyco-berbère. Si l'arabe a transmis en l'adaptant cette toponymie, il faut bien admettre que celle-ci fut apprise et reprise de populations romanes: le latin, le roman étaient donc indigènes, bien audelà des villes ${ }^{8}$

Enfin, la réduction et disparition de cette Romania primitive s'est nécessairement étalée sur plusieurs siècles. La rareté des matériaux ne permet pas de fixer la mort proprement dite. Jusqu'au XIIème siècle au moins des communautés chrétiennes sont régulièrement signalées par des hommes de lettres arabes. La plupart ne mentionnent, c'est assez naturel, que la chrétienté de ces groupes (donc l'aspect cultuel), groupes de moins en moins nombreux. Certains, comme El Idrisi (XII ${ }^{\mathrm{ème}}$ siècle), attestent directement la langue ${ }^{9}$. Cet auteur nous dit en effet qu'à son époque on parle encore le latin d'Afrique à Gafsa (Tunisie). Comme chez d'autres écrivains arabes, ces chrétiens sont qualifiés d'Afariqs (Africains) ou descendants directs de l'Afrique romaine.

Ce type de témoignage rejoint les travaux d'archéologues et d'historiens. Ainsi Amor Mahjoubi (1966) a-t-il montré qu'une communauté chrétienne existait à Kairouan (Tunisie) au XI ${ }^{\mathrm{e} m e}$ siècle et qu'elle avait sa propre épigraphie. Avant lui, Charles Courtois s'était également consacré à l'étude de ces communautés chrétiennes du Moyen Age ${ }^{10}$

Plus tard encore des communautés chrétiennes semblent s'être maintenues, par exemple dans le Nefzaoua tunisien jusqu'au XIV ${ }^{\text {ème }}$ siècle et à Tozeur (Tunisie) jusqu'au XVIII ${ }^{\text {ème }}$ siècle $^{11}$.

Compte-tenu des observations précédentes, on voit mal de tels groupes se maintenir chrétiens et évacuer toute pratique romane en s'arabisant en bloc mais sans rien prendre de la nouvelle religion. Ce schéma semble très irréaliste sur le plan du fonctionnement des identités. Une hypothèse plus vraisemblable est que dans une partie au moins de leurs pratiques linguistiques, ces groupes étaient

\footnotetext{
${ }^{8}$ Voir en Tunisie des évolutions comme UTHINA $>O U D N A$, CHUSIRA $>K E S R A$ (La Kessera), KLLPEA $>K L I B Y A$ (Kélibia). D'après les traits évolutifs ici repérables (maintien des voyelles atones finales, sonorisation des occlusives intervocaliques, syncopes des voyelles intermédiaires etc.), on peut imaginer une Romania africaine en préparation très proche de la Romania occidentale, en particulier de l'Ibéro-roman.

${ }^{9}$ El Idrisi ou Al Idrisi, Le Maghreb au VI ${ }^{\text {ème }}$ siècle de l'Hégire (XII ${ }^{\text {ème }}$ siècle ap. J.-C.). Différentes éditions, dont Publisud-OPU (1983) ici consultée.

${ }^{10}$ Voir Amor Mahjoubi, Nouveau témoignage épigraphique sur la communauté chrétienne de Kairouan au XI ime siècle (AFRICA-I, 1966) et Charles Courtois, "Grégoire VII et l'Afrique du Nord", (Revue historique, 1945). Voir surtout Hady Roger Idris, La Berbérie orientale sous les Zirides (Adrien-Maisonneuve, 1962), tome second, pp. 757 et suivantes.

${ }^{11}$ H.-R. Idris, ouv. cité, p. 761.
} 
restés romanophones, s'arabisant progressivement pour s'adapter à la société globale. Le roman d'Afrique se serait donc confidentialisé toujours plus au cours du Moyen Age et par la suite, fonctionnant en circuit fermé et s'appauvrissant comme une langue de l'intimité et du culte.

On constate qu'il est relativement facile de suivre la restriction du christianisme maghrébin. On a nettement moins de preuves concernant les lectes romans que pouvaient utiliser ces communautés. Mais une correspondance générale de principe existe entre les deux ordres, et ce qui est avéré pour l'un doit l'être en bonne partie pour l'autre. Comme la langue arabe elle-même l'a consacré, être chrétien en Afrique c'est être "romain" ${ }^{12}$. Préserver cette identité de romainchrétien ou d'africain relevait on le comprend du tour de force. S'arabiser c'était en revanche se donner les moyens de progresser dans la société arabo-islamique qui se mettait en place. On voit bien différents chrétiens de bonnes familles accéder à des fonctions importantes du $\mathrm{X}^{\text {ème }}$ au $\mathrm{XII}^{\mathrm{ème}}$ siècle par exemple ${ }^{13}$, mais ces cas, multiples dans une période initiale de relative tolérance, se raréfient par la suite. Il y a là en effet une anomalie fondamentale, liée au fait que le pôle roman qui s'affaissait, fonctionnait au fond comme le pôle arabe du Maghreb qui montait. Dans chacun en effet langue et culte étaient intimement associées au point d'être vus comme deux éléments constitutifs indispensables d'une identification harmonieuse. Aussi le statut de Chrétien arabophone restait-il par définition provisoire et les conversions étaient-elles nombreuses et radicales. On abandonnait ainsi une identité homogène pour une autre identité également homogène, mais de plus majoritaire et donc socialement porteuse.

Ce schéma d'évolution sociolinguistique et identitaire, dut comme toujours être d'abord le fait des classes citadines et les plus élevées de la population autochtone. Pour des motifs de reproduction de leur pouvoir, la solution devint rapidement l'arabisation-islamisation (dans cet ordre). On peut penser que de proche en proche, toutes les communautés chrétiennes furent ensuite touchées, en sens inverse de la pyramide sociale et en allant des villes vers les campagnes. Aussi les chrétiens ruraux furent-ils sans doute affectés plus tard par le changement, comme le montrent les localisations géographiques des isolats au cours du Moyen-Age, pour finir situés sur les marges désertiques du Maghreb oriental.

On ajoutera qu'une tare fondamentale dévalorisait le pôle roman. Les variétés romanes qu'on imagine se restreignant statutairement et fonctionnellement, ne disposaient désormais plus d'un vrai support référentiel écrit et normatif. On tentait de reproduire dans un dénuement presque total, et de moins en moins bien, des normes provenant d'une autre époque et d'autres classes sociales. D'autre part, le temps passant, les groupes chrétiens ou romans se dispersaient davantage en îlots, noyés dans les continuum berbère ou arabe. Aucune chance donc que les systèmes oraux romans subsistant éventuellement dans ces communautés condamnées pussent lutter à armes égales avec l'arabe. Celui-ci, riche de sa littérature, de ses normes et de ses fonctions sociales

\footnotetext{
${ }^{12}$ En Afrique du Nord, l'appellatif roumi, littéralement "romain", désigne toujours le chrétien et, par extension, l'européen.

${ }^{13}$ Voir toujours H.-R. Idris et A. Mahjoubi sur ce point, ouv. cités.
} 
terrassait statutairement la latinité d'Afrique comme le berbère dans les campagnes.

2-1-b. La deuxième Romania, avant le $\mathrm{XIX}^{\text {ème }}$ siècle.

On désignera ainsi l'ensemble des implantations chrétiennes et romanes directement issues d'Europe cette fois, non autochtones donc. Les deux composantes, européenne et autochtone, avaient peu de chances de se rencontrer durablement, $\mathrm{du}$ fait que les implantations européennes restaient fondamentalement côtières au moment où les communautés autochtones se dispersaient et reculaient comme on vient de le voir. Ce croisement se produisit toutefois sur les côtes de la Tunisie, du fait de la protection normande de Sicile $\left(\mathrm{XII}^{\text {ème }}\right.$ siècle). Des communautés autochtones se réfugièrent ainsi dans certains ports contrôlés, où s'installaient en même temps des chrétiens venus de Sicile. Mais, le plus souvent, la séparation est profonde: une Romania s'éteint au moment où une autre s'implante.

Cette deuxième manifestation historique du pôle roman relaye en effet à peu près parfaitement la Romania primitive. C'est une période d'affirmation des nations romanes, de leurs mouvances commerciales et des impérialismes naissants. Les mieux placés sont au départ les Ibéro-romans et les Italo-romans. La proximité les conduit très tôt à prendre pied sur les côtes du Maghreb, soit par relations commerciales, soit militairement dans le prolongement des "reconquêtes" péninsulaires sur les musulmans. Très tôt sont également présents les ports du Golfe du Lion.

On voit les Génois, les Pisans, les Florentins, les Catalans ou Aragonais, les Provençaux, commercer activement avec l'Afrique du Nord entre le $\mathrm{XI}^{\text {ème }}$ et le $\mathrm{XV}^{\text {ème }}$ siècle, alors même qu'une bonne partie de l'Europe méridionale est encore arabo-musulmane.

Se dessinent ainsi quelques zones préférentielles. Les Génois et les Provençaux sont présents à Ceuta(Maroc) et Bougie(Algérie) où ils ont des consulats permanents aux XII ${ }^{\text {ème }}$ et $\mathrm{XIII}^{\text {ème }}$ siècles. Pisans et Vénitiens, en concurrence avec les précédents, paraissent mieux implantés en Tunisie ${ }^{14}$.

Par le biais de comptoirs actifs s'installe donc une Romania côtière qui apporte directement des idiomes romans régionaux (comme le catalan, le provençal, le vénitien), des variétés intermédiaires véhiculaires conçues pour le commerce avec les maghrébins.

C'est à ce moment que se mettent en place les grands Etats romans. Aussi les Marseillais jouent-ils pour leur compte dans un premier temps et pour la France ensuite (à partir du XV ${ }^{\text {ème }}$ siècle) un rôle toujours plus important. En plus de Ceuta et Bougie, on les voit au $\mathrm{XIII}^{\text {ème }}$ siècle à Oran, Tlemcen (Algérie), à Tunis. Dans cette partie orientale du Maghreb, l'influence de la France méridionale ne fera que croître. Ainsi en 1520, les Provençaux obtiennent-ils le privilège de la pêche du corail de Bône (Algérie) à Tabarka (Tunisie). Ce privilège, de proche en proche, sera reconduit jusqu'à l'époque coloniale.

\footnotetext{
${ }^{14}$ Voir sur ce point les travaux anciens de De Mas Latrie, particulièrement Relations et commerce de l'Afrique septentrionale ou Magreb avec les nations chrétiennes au Moyen Age (Paris, FirminDidot, 1886).
} 
A l'ouest, les Portugais s'installent à Ceuta en 1415, inaugurant ainsi une présence politique ibéro-romane qui dure encore. Ils s'implantent plus ou moins durablement dans quelques cités côtières du Maroc. Les Espagnols se couleront souvent dans cette mouvance préalable des Portugais, ou celle des CatalansAragonais à partir de la réunion des couronnes d'Aragon et de Castille en 1479.

\section{siècles).}

2-1-c. La troisième Romania et la montée du français $\left(X X^{\text {ème }}-X^{\text {ème }}\right.$

$\mathrm{Au}$ moment où la France va intervenir directement en Algérie (1830), deux zones d'influence des langues romanes (autres que le français) se dessinent avec netteté.

A l'ouest, une zone d'influence espagnole, du Maroc au littoral central d'Algérie. L'Espagne dispose d'implantations sous forme de comptoirs, colonies militarisées ou "presidios". La colonisation française de l'Algérie contribue indirectement à accroître cette pression occidentale de l'Espagne qui intervient au delà des presidios, par exemple en prenant Tétouan (Maroc) en 1860. Cette puissance revendique ainsi sa propre zone coloniale dans le nord du Maroc et l'obtiendra bientôt, par les accords d'Algésiras en 1906 et le protectorat effectif de 1912 à 1956. Au Sud, le Sahara espagnol sera protectorat jusqu'en 1975. Indirectement, la colonisation française de l'Algérie favorise l'extension de l'aire d'influence "naturelle" des parlers ibéro-romans. L'Algérie occidentale (on pense à l'Oranie) accueille des flux très importants de petits colons espagnols (andalous, valenciens, catalans) dont la langue première ne sera pas le français dans l'immédiat.

A l'est, une zone d'influence italo-romane ancienne allait de l'est algérien aux côtes de Tunisie. Pêche, récolte d'éponges et de corail, investissements industriels divers avaient semble-t-il fixé nombre d'immigrants avant même le protectorat français. Mais la colonisation italienne contrecarrée par la France en Tunisie prit forme en Libye à la suite de la guerre italo-turque de 1911-1912, ce pays étant annexé jusqu'à la seconde guerre mondiale.

Ces deux mouvances, espagnole à l'ouest, italienne à l'est, tout en complétant une forme d'acculturation romane globale, constituaient pourtant des obstacles à la logique propre de la France et du français, celle-ci tendant à capter à son profit l'ensemble du pôle roman. La progression du français, suivant les modalités internes des deux autres mouvances, adopta des stratégies et des profils différents.

Il faut à nouveau repartir des faits historiques. Une absence d'intervention politique de la France vers le Maroc et la Tunisie à partir de ses positions algériennes, eût pu laisser les mains libres à l'Espagne et surtout, à l'autre bout, favoriser une implantation plus profonde et durable de l'italien en Tunisie. Car le point chaud est avant tout la Tunisie. A propos de cette portion du Maghreb une thèse s'affirme durant la seconde moitié du XIXème siècle, celle de la continuité naturelle Algérie-Tunisie, très clairement revendiquée en 1880 :

"[.] En un mot, quelques heures de marche sur le même littoral africain suffisent pour entrer de plain-pied dans l'immobile Orient des siècles passés, après avoir franchi le seuil de cet autre Orient moderne, orné de tous les 
prodiges de la civilisation européenne. Sans doute, le temps ne peut manquer de faire justice de cette choquante anomalie, et la Tunisie, qui sous tous les rapports, n'est guère que la continuation et même le complément nécessaire de l'Algérie, doit être un jour rattachée à cette dernière en réparant ainsi les profondes blessures que lui a infligées cette séparation contre nature. C'est une question d'humanité, mais c'est aussi une question d'intérêts français. ${ }^{15}$.

L'entrée en Tunisie se fera en 1881. Le point de départ officiel est le comportement agressif de tribus tunisiennes frontalières de l'Algérie française, des incursions s'étant produites en début d'année 1881 en Khroumirie, zone montagnarde de contact entre les deux pays. Une intervention militaire française à la fin d'avril conduira rapidement au Traité dit du Bardo (12 mai 1881) plaçant la Tunisie sous le Protectorat de la France.

Quels sont ces intérêts?

Des intérêts économiques bien évidemment. Une circulaire du Ministère français des Affaires Etrangères (9 mai 1881) estime que dans la dette tunisienne les fonds français représentent les trois cinquièmes, la France ayant contribué depuis plusieurs années à l'installation des postes, du télégraphe, du chemin de fer, à différents travaux d'aménagement portuaire. Sur le terrain, compagnies italiennes et françaises sont en concurrence directe

Mais aussi des intérêts culturels et linguistiques. Les observateurs français sont conscients de l'excellente implantation des italiens. Les représentants de cette "Nation" sont bien écoutés par les autorités tunisiennes, une véritable infrastructure culturelle italienne est en place: écoles et collèges à La Goulette, Tunis, Sousse, Sfax, école professionnelle à Tunis. Et l'on comprend très vite qu'une telle communauté italienne, anciennement implantée et très dynamique, pourrait bientôt profiter du Protectorat français pour étendre sa propre influence. Il appartiendra donc à l'Instruction publique française d'étouffer à terme la langue romane concurrente en la privant d'assises statutaires et en dialectalisant-minorant toujours plus les communautés d'origine italienne.

Quelques constats et analyses de l'époque confirment ce point de vue.

"Avant l'occupation française, l'enseignement français était presque nul dans la Régence; notre langue était supplantée par l'italien, et l'arabe était seul usité dans les écoles de Koran, et les medraças des villes. ${ }^{\prime 16}$.

"La France aura les plus grands efforts à faire pour maintenir son influence intellectuelle dans le pays et pour la défendre contre les rapides progrès d'une nation entreprenante [L'Italie] qui profite habilement de toutes nos hésitations et de toutes nos lenteurs administratives. "17 niveaux

Avec l'Espagne, une trentaine d'années plus tard, le problème était à deux

D'une part, dans son protectorat, cet Etat engageait avec succès sa propre politique linguistique. Ainsi tendait à naître vers le milieu du $X X X^{\text {ème }}$ siècle une véritable hispanophonie du Maghreb que la France ne pouvait guère contrecarrer. Le faux paradoxe est que cette potentialité fut étouffée par la généralisation

\footnotetext{
${ }^{15} \mathrm{P}$. de Tchihatchef, Espagne, Algérie, Tunisie. Paris, 1880.

${ }^{16} \mathrm{~L}$. Lanier, L'Afrique, choix de lectures de géographie. Paris, Belin, 1889. p. 337.

${ }^{17} \mathrm{P}$. Foncin, L'enseignement en Tunisie (1882), cité par L. Lanier.
} 
dynamique du français au niveau de l'Etat marocain à partir de l'indépendance, non par la France elle-même.

D'autre part le français se trouvait en contact direct avec différents lectes ibéro-romans oraux, mais en Algérie. Variétés populaires et dialectales de l'espagnol (andalou) ou du catalan (valencien), compte-tenu des principales provenances des ouvriers espagnols utilisés par la colonisation française. Ces locuteurs transportaient leur propre diglossie d'Espagne (par exemple le valencien ou le mahonnais [Baléares, Minorque] dominés par l'espagnol), mais la versèrent aussi dans un nouveau rapport de force. Cette fois l'espagnol et les "dialectes" d'Espagne étaient statutairement dominés par le français. L'absence d'une quelconque assise scolaire (contrairement au cas de l'italien en Tunisie) et le recrutement très populaire des employés et petits colons espagnols infériorisaient l'ibéro-roman sous toutes ses formes. Mais en revanche le poids humain des communautés ainsi déplacées, leur natalité forte et leurs comportements grégaires mettaient en difficulté relative le français dans l'ouest de l'Algérie. La colonisation française devait traiter le problème d'une véritable petite Espagne en territoire français. C'est comme toujours à l'Instruction Publique et à une aspiration par le haut que devait revenir l'essentiel de la francisation ultérieure.

\section{2-2. Bilan intermédiaire: constance du pôle roman et structure tripolaire.}

A l'échelle de l'Histoire profonde il apparaît donc bien qu'une caractéristique fondamentale du paysage sociolinguistique maghrébin est celle d'être triadique. Trois pôles linguistiques sont en présence depuis l'Antiquité, et les pratiques linguistiques comme les traits identitaires sont constamment conditionnés par ces axes d'attraction-répulsion. Aujourd'hui comme hier, les Maghrébins construisent leur personnalité collective par rapport à ces trois pôles: berbère, roman, arabe, dans l'ordre historique d'apparition.

Nous avons consacré un long développement au pôle roman, car celui-ci est le plus souvent mésestimé, réduit à une pure superficie, assimilé régulièrement à une famille exotique, non maghrébine. Ce regard mérite d'être corrigé tout d'abord par rapport à l'Histoire globale mais aussi parce qu'en réduisant ainsi on perd en force heuristique. Comme on vient de le voir, les faits plaident pour une constance de ce pôle, certes à travers des configurations variables. Tantôt le pôle est statutairement dominant mais souvent statistiquement dominé sur le terrain (cas du latin au début de l'ère chrétienne, du français depuis le $\mathrm{XIX}^{\mathrm{ème}}$ siècle). Tantôt le pôle est statutairement dominé, ségrégé, dialectalisé, mais en même temps tenace dans le recul. C'est par exemple la longue période de régression médiévale de la Romania primitive, période sur laquelle nous sommes forcément mal renseignés. En effet, les pratiques de communautés qui meurent n'existent pas ou ne sont pas répercutées dans une civilisation de l'écrit. Mais en dépit de ce recul le pôle est toujours là dans les faits, dans la familiarité relative, dans l'imaginaire collectif. Les Gafsiens dont nous entretient El Idrisi parlaient-ils latin? Vraisemblablement pas, plutôt une variété romane encore perçue comme du latin par les arabophones de ce temps. Dans tous les cas, la présence du pôle, pour minime, incomplète ou 
infériorisée qu'elle puisse paraître, a, parmi diverses conséquences, celle de limiter la propension expansive des autres pôles.

Ceci vaut pour le pôle roman, face au libyque et au punique (Antiquité), puis au berbère et à l'arabe. Mais ceci vaut également pour les deux autres pôles, car chacun, à des époques variables, résiste aux pressions des deux autres. Il y a donc aussi le berbère face à l'arabe et au roman, l'arabe face au berbère et au roman.

\section{Deuxième partie: principes dynamiques et comportements systémiques}

\section{Principes fonctionnels et dynamiques du système tripolaire.}

\section{3-1. Deux principes complémentaires.}

\section{3-1-a. Principe d'opposition et d'alliance.}

L'un des pôles peut sembler s'opposer directement à un deuxième (par exemple le français opposé à l'arabe depuis la colonisation). Il s'oppose en fait aux deux autres, tout en se nourrissant partiellement d'une relation positive avec le troisième (par exemple la relation préférentielle français-berbère durant la même période).

De même le berbère semble s'opposer directement à l'arabe (revendication ethnolinguistique), mais sa relation précédente avec le français n'est que conjoncturelle, tactique en somme, comme elle le fut sans doute avec le latin face au punique et avec le punique face au latin.

Enfin l'arabe, aujourd'hui langue d'Etat, s'oppose tantôt au français en tendant à impliquer le pôle berbère dans ce rejet (thèse de l'étranger et de la langue coloniale), tantôt au berbère en se solidarisant implicitement avec le français langue "internationale" ou de culture écrite (thème du patois berbère, oral et archaïque).

On comprend qu'un système aussi souple permet la survie de chaque pôle, et empêche par principe toute évolution radicale du paysage linguistique global.

\section{3-1-b. De l'homogénéité à la dialectalisation.}

Chacun des pôles semble devoir passer par des phases de fragmentation qui sont aussi des phases d'oralisation et de dialectalisation (= phase -), ou au contraire des phases de centralisation.

Il semblerait que les phases du premier type affaiblissent la résistance du pôle (sans l'éradiquer pour autant), tandis que les phases du deuxième type développent au maximum les potentialités sociolinguistiques totalitaires du pôle en question $(=$ phase +$)$.

Tout se passe en fait comme si chacun des pôles changeait de niche statutaire de référence pour assurer son développement. Ces niches statutaires sont 
en fait des rôles assumés par les langues et les pôles. Ils semblent être au nombre de trois :

Rôle 1 : fonction mondiale (= propension à l'universalité)

Rôle 2 : fonction nationale (= l'Etat, le système politique global)

Rôle 3 : fonction locale (= le village, la région, les activités ordinaires).

On peut discuter à l'infini de la signification "objective" de ces trois grands rôles. Observons simplement qu'ils nous obligent à sortir de considérations strictement linguistiques, en admettant une macro-classification d'identification collective des groupes, assumée directement par ces mêmes groupes.

Est alors strictement local (rôle 3) ce qui permet aux individus de s'intégrer dans le schéma de reproduction sociale, dans les activités économiques et rituelles des communautés maghrébines (le plus souvent rurales). C'est le cas typique du berbère au Maroc et en Algérie de nos jours, mais c'est aussi le cas de l'arabe dialectal dans toutes les zones désormais arabophones.

Le cadre de la nation (rôle 2) est ici un cadre de référence moderne, celui des Etats maghrébins et de leurs mouvances territoriales. Naturellement, en remontant dans le temps ce cadre est difficilement utilisable (mais il y a des propensions nationales comme dans l'Afrique latine ou certaines dynasties araboberbères : Almoravides, Mérinides etc.). On peut toutefois le conserver en évitant deux écueils. Le premier serait d'admettre une équivalence nation $=$ ethnie. Dans leur dynamique identitaire les groupes ethniques franchissent souvent le pas (ce qui d'un point de vue "militant" peut être considéré comme acceptable), mais il ne saurait y avoir en termes appropriés de nation berbère ou de nation arabe, à plus forte raison de nation romane dans l'Histoire du Maghreb. En deuxième lieu la nation n'est pas la umma ou "nation" arabo-musulmane. Ce principe d'identification collective est d'un autre ordre, spirituel, et renverrait plutôt au rôle international mais sans donner les garanties d'internationalité telles qu'on les conçoit aujourd'hui. Comme le soulignait en 1979 Gilbert Grandguillaume "l'idée de nation au sens moderne -parfois traduite en arabe watan- est étrangère à la tradition politique arabe [.]"18.

Avec le niveau international (rôle 1), on quitte le plan strictement maghrébin pour considérer la véhicularité proprement internationale et mondiale d'un pôle ou d'une langue. Bien sûr, la mondialité est elle-même relative, toujours discutable, et on aura toujours intérêt à la mesurer à l'étalon des jugements de locuteurs.

Pour les maghrébins de l'Antiquité, le monde c'est la Méditerranée plus quelques zones lointaines dont les plus commerçants ou les plus instruits entendent parfois parler. Dans ce cadre sont associés au rôle 1 le grec, le punique, le latin

\footnotetext{
${ }^{18} \mathrm{G}$. Grandguillaume, Langue, identité et culture nationale au Maghreb. Peuples méditerranéens, 1979. Repris dans Language in Tunisia (Tunis, Bourguiba Institute of Modern Languages, 1983).
} 
Pour leurs successeurs médiévaux, le monde a changé de configuration. Le rôle 1 revient par exemple aux langues romanes voire à l'arabe, mais celui-ci semble déjà coller davantage au rôle d'identification nationale.

Aujourd'hui le monde est plus vaste encore. C'est d'une part le prolongement arabo-islamique, donc l'arabe, mais pas dans la variété locale. C'est d'autre part l'Europe et le monde dit "occidental", mais un monde qui n'est pas ailleurs seulement, car le Maghreb y participe directement. Pour l'instant, le vecteur de cette intégration planétaire est le français, immédiatement avant l'anglais

\section{3-1-c. Applications au paysage linguistique d'Afrique du Nord.}

Le pôle berbère semble être passé par une phase (+) durant la plus haute antiquité. C'est d'ailleurs aujourd'hui encore le fondement de la revendication d'écriture du berbère (orthographe tifinagh), qui crispe tant le pôle arabe depuis quelques dizaines d'années ${ }^{19}$. Le pôle roman et le pôle arabe l'ont ensuite placé dans la situation d'un idiome de la ruralité, relégué et atomisé à travers le Maghreb (la vraie "langue" de référence étant dans ce cas le latin, l'arabe ou le français). Son émiettement en nébuleuse de parlers aux $\mathrm{XIX}^{\text {ème }}$ et $\mathrm{XX}^{\mathrm{ème}}$ siècles le situe bien dans le rôle 3 .

Le cas du latin au sein du pôle roman est à nouveau exemplaire car il permet de contrôler la logique globale du système sociolinguistique observé. Le latin est en phase $(+)$ rôle $l$ durant sa période d'implantation (avant et aux environs de l'ère chrétienne). Son statut global est alors très fort (langue du pouvoir militaire et administratif, langue de l'écrit, de la littérature etc.) mais son implantation est insuffisante pour impliquer sa survie à l'échelle de la région. En revanche, durant la phase suivante (premiers siècles de l'ère), il amorce une dialectalisation et rejoint le rôle 2 : il est désormais une langue régionale encore relativement haute ou centrale (reconnue comme telle par ses locuteurs), mais c'est tout de même le latin d'Afrique. Sans doute est-ce la période ou cette langue est la plus "menaçante" pour le reste du paysage linguistique, car devenue autochtone et encore réputée universelle (cf. sa liaison à la communauté de culte). En vérité, il y avait peu de risques d'exclusivité du latin dès lors que le berbère assurait en gros à ce moment les rôles 2 et 3 (une partie revenant semble-t-il au punique).

L'examen du pôle arabe confirme la mécanique du système. L'arabe arrive principalement avec le rôle 1. Il concurrence directement le latin et le supplante, car comme celui-ci il représente le pouvoir, l'écrit et la littérature, l'identité religieuse enfin. C'est probablement cette concordance de faisceaux fonctionnels sociolinguistiques qui explique le recul relativement massif du pôle roman primitif. Ce dernier est donc progressivement rejeté vers le rôle 3 (phase d'extinction) qu'il tend ainsi à partager avec le berbère.

\footnotetext{
${ }^{19}$ En 1981, la revue marocaine Amazigh (=Berbère), dirigée par Mahjoubi Aherdan, signalait la prochaine mise à disposition des premières machines à écrire à clavier berbère. L'été suivant les machines étaient saisies et la revue interrompue. Plus près de nous, le ler mai 1994, lors d'une manifestation organisée par l'association berbériste Tilelli à Errachidia (banderoles en français et en berbère), c'est d'abord le retrait des banderoles en berbère qui fut demandé par les autorités, avant que quelques responsables de l'association ne soient arrêtés, jugés, condamnés pour certains, puis finalement amnistiés (rapport du journal "Le Monde").
} 
Car en effet l'arabe prétend de plus en plus par la suite au rôle 2. Il le peut d'autant mieux que lui-même entre à son tour dans le processus de dialectalisation. Mais le paradoxe expérimenté par les autres pôles se reproduit ici : d'une part l'arabe adhère de plus en plus au terrain (rôles 2 et 3), mais d'autre part ce mouvement de légitimation "locale" l'éloigne proportionnellement du rôle 1. Bien entendu, ce paradoxe apparent est très cohérent dans le système observé. Aucun des pôles en présence ne peut à lui seul assumer les trois rôles : cela n'a jamais été possible en vingt siècles d'Histoire et a fort peu de chances de se réaliser dans un avenir proche.

Le pôle roman moderne sous ses formes antérieures au XIX ${ }^{\text {ème }}$ siècle, ou sous la forme du français à époque contemporaine, se manifeste également au départ dans le rôle 1. Les langues romanes comme l'espagnol, l'italien, le catalan et le provençal à un moindre degré, ont eu dans les circuits portuaires et commerciaux un statut de langue dominante. On peut d'une part le présumer, mais aussi l'établir en remarquant le poids de ces langues (les deux premières surtout) dans les variétés intermédiaires produites du $\mathrm{XV}^{\text {ème }}$ au $\mathrm{XIX}^{\mathrm{ème}}$ siècles, la lingua franca en premier lieu ${ }^{20}$.

Dans le cas du français, le processus de dialectalisation s'était nettement mis en marche avec la formation du (ou des) français des Pieds-Noirs, processus que l'on a cru interrompu par la décolonisation et l'exode massif des Français du Maghreb. Le dépôt local du français s'est en fait poursuivi autrement, et de nombreux observateurs constatent aujourd'hui différentes formes d'appropriation, en Algérie tout particulièrement ${ }^{21}$.

On reconnaît derrière ce processus de dialectalisation la trame de la diglossie. Diglossies avérées: celles de l'arabe succédant à celle du latin, ou éléments d'une diglossie potentielle du français. A chaque fois se produit une cassure interne du pôle considéré, ce qui renforce le pôle par certains aspects (l'enracinement sur le terrain) et l'affaiblit par d'autres (perte d'homogénéité statutaire).

Pour finir, on observera que le mouvement inverse est possible, mais semble-t-il plus rare. Une tendance à la centralisation-homogénéisation est à l'oeuvre dans les pôles arabe et berbère, mais cette tendance, dans l'un et l'autre cas n'a pas le même point de départ. L'arabe a bien identifié sa diglossie, depuis les Indépendances en particulier, et l'on part de ce constat pour tendre, dans la mesure du possible, vers un arabe moyen (cf. les appellations "arabe classique simplifié", "arabe intermédiaire" etc.).

\footnotetext{
${ }^{20}$ La lingua franca ou langue intermédiaire à base romane simplifiée paraît bien attestée aux XVIIème et XVIIIème siècles. Ainsi en 1612, Fray Diego de Haedo la donne-t-il pour universelle à Alger (Topografia e historia general de Argel). On la retrouve également en Algérie et Tunisie au début du XIX ${ }^{\text {eme }}$ siècle, mais elle se modifie alors rapidement au contact du français. Hugo Schuchardt s'y est intéressé au début du siècle ("Die Lingua Franca", Zeitschrift für Romanische Philologie, 1909). Voir le point de Pierre Perego, Les sabirs (Encyclopédie Le Langage, Gallimard-La Pléiade, 1968). Plus récent, voir également Paul Siblot, De l'opprobre à la glorification...Le cas des sabirs d'Afrique du Nord (Cahiers de linguistique sociale, 1993).

${ }^{21}$ Voir différents travaux de linguistes algériens sur les particularismes lexico-sémantiques dans le cadre du colloque Le français au Maghreb (Aix-en-Provence, septembre 1994). A paraître fin 1995.
} 
On cherche donc en théorie à colmater la brèche en produisant un intermédiaire accessible au plus grand nombre. Dans le cas du berbère en cette fin de $\mathrm{XX}^{\mathrm{ème}}$ siècle, on part d'une langue fortement éclatée et se construit alors un mouvement de production d'une variété supra-locale écrite, une langue qui pourrait sortir à terme du rôle 3 (local) pour tendre vers le rôle 2 (national voire maghrébin $)^{22}$.

\section{3-2. Bilan: tendances lourdes contemporaines.}

\section{3-2-a. Interactions et système tripolaire du XIX ${ }^{\text {ème }}$ siècle aux Indépendances.}

Il ne peut être question, dans ce cadre limité, de revenir sur l'ensemble des problématiques sociolinguistiques depuis la colonisation française, du moins peuton en rappeler les grands axes ${ }^{23}$.

Ainsi que nous l'avons souligné, il n'y avait pas à proprement parler "irruption" du français. Dans l'esprit d'une bonne part des acteurs européens, la "continuité romane" fut d'ailleurs reprise comme un argument de colonisation. Mais sur un plan plutôt culturel, car on estimait que la France avait une mission historique, celle de reprendre le flambeau de Rome: celle-ci avait amené la civilisation avec sa langue, la France devrait donc en faire autant mais avec sa propre langue, celle des lumières et de la modernité. Ceci est typique d'une vision très positive du français et partant négative pour les autres manifestations de la Romania. Egalement typique de la philosophie de la langue nationale et de l'Instruction publique en France au XIX ${ }^{\text {ème }}$ siècle.

A partir de là, il était logique que le français affrontât directement le pôle arabe, dès lors que celui-ci assumait depuis quelques siècles le rôle national. Un rôle qui devait lui être contesté puisque la France entendait fonder une nouvelle nation. S'en suivirent une politique de division et de minoration du pôle arabe, dans un moindre degré du pôle berbère (celui-ci moins dangereux et pouvant servir le français). Très tôt en effet, les observateurs français insistent sur l'écart existant entre les pratiques locales de l'arabe (arabes dialectaux, oraux, paysans etc.) et l'existence d'une variété haute mais claquemurée, l'arabe dit "du Koran", impropre à fonder une quelconque nation moderne ${ }^{24}$. Divisé, l'arabe est -on le voit- réduit et largement caricaturé par la colonisation française, réputé impuissant face au Français. Faut-il souligner que cette dichotomie pèse jusqu'à nos jours?

Pour la colonisation et le français, l'autre pôle, le berbère, présente un intérêt évident. Sur le plan statutaire officiel, cette langue est certes minorée, parce que locale et non comparable ni à l'arabe, ni au français, à plus forte raison. Mais sur

\footnotetext{
${ }^{22}$ Pour la question du berbère et de sa graphie, voir en particulier Salem Chaker, "Langue et culture berbères en Algérie depuis 1988" (Cahiers de linguistique sociale, 1993 : Minoration linguistique au Maghreb).

${ }^{23}$ Outre différents ouvrages cités en notes, voir Francis Manzano, "Des maghrébins lettrés observent le paysage linguistique d'Afrique du Nord" (Cahiers de linguistique sociale, 1993 : Minoration linguistique au Maghreb), "La francophonie dans le paysage linguistique du Maghreb : contacts, ruptures et problématique de l'identité" (Aix-en-Provence, 1994, cf. note 19).

${ }^{24}$ Qualification constante dans les textes français du XIX ${ }^{\text {ème }}$ siècle.
} 
le plan culturel et historique, le pôle berbère représente l'antériorité à l'arabe, ce qui prive en quelque sorte l'arabe de "droit historique" sur le Maghreb. Se met donc en place chez les européens un mouvement de description anthropologique et linguistique du berbère ${ }^{25}$. Et dans ce dossier s'en ouvre un autre, celui de "l'authenticité", de la différence berbère, voire de "l'européanité" berbère. Ainsi l'anthropologie physique soulignera-t-elle régulièrement la présence d'un stock d'origine alpine européenne, et nombre d'études établiront des rapprochements avec les populations des Alpes, des Pyrénées, du Massif Central ou de la Bretagne. On souligne également la chrétienté des berbères de l'antiquité [en fait d'une partie des berbères] ou leur judaïcité qui, indirectement, peuvent servir la cause française et francophone. En dépit de difficultés disciplinaires réelles, on tend donc à établir l'existence d'une race différente, d'une culture et d'un passé différents, correspondant bien entendu à une langue différente ${ }^{26}$. Se crée ainsi un double stéréotype : il suffit au Maghreb de se baisser pour découvrir des témoignages de la romanité antique, il suffit aussi de se pencher sur la culture berbère pour voir qu'elle est pétrie de reliques païennes et chrétiennes.

Cette approche identificatrice directe du berbère et indirecte de l'arabe est certes typique de l'époque et du projet colonial, mais elle nous montre surtout à quel point les choix linguistiques et les jugements identitaires sont étroitement conditionnés par le modèle tripolaire. Il s'agit ici d'une série de manifestations d'une relation entre pôle roman (français) et pôle berbère. Le premier organise une jonction avec le second pour diminuer la force du troisième (le pôle arabe). Le pôle berbère "sollicité" ne renvoie pas forcément la réponse espérée, par exemple sur la question religieuse, bien que certaines composantes minoritaires aient pu être "rechristianisées" en Algérie ${ }^{27}$. Il trouve pourtant satisfaction dans le fait que la colonisation en Algérie et le protectorat au Maroc donnent un véritable statut aux études berbères, non seulement au niveau universitaire le plus haut (par exemple aux "Hautes Etudes Marocaines") mais aussi dans les études secondaires et la formation des cadres (comme le Collège Berbère d'Azrou, expérience qui

\footnotetext{
${ }^{25}$ Durant la seconde moitié du XIX ${ }^{\text {ème }}$ siècle, de nombreux ouvrages ou articles s'intéressent à la matière berbère et plus précisément à la composante kabyle d'Algérie, en soulignant régulièrement la dualité algérienne. Ainsi H. Aucapitaine, Etudes sur le passé et l'avenir des Kabyles (Paris, 1864), Cdt. Duhousset, "Les races algériennes, les Kabyles du Djurjura" (Revue des cours scientifiques, 1868), D. Kaltbrunner, Recherches sur l'origine des Kabyles (Genève, 1871), E. Guimet, Arabes et Kabyles, pasteurs et agriculteurs (Lyon, 1873), J. Dugas, La Kabylie et le peuple kabyle (Paris, 1878) etc. De tous ces ouvrages, l'un des plus justement célèbres reste celui de L.-J. Hanoteau \& M. Letourneux, La Kabylie et les coutumes Kabyles (Paris, 1873). Hanoteau avait donné précédemment des ouvrages sur la grammaire du Kabyle (1858) et du Tamacheq (1860). Cette première génération de travaux a directement précédé les études proprement dialectologiques et comparatistes comme celle de René Basset à la fin du XIX ${ }^{\text {ème }}$ siècle.

${ }^{26} \mathrm{On}$ se garde bien toutefois d'un regard entièrement positif sur les berbères, souvent présentés comme un ensemble appauvri et largement corrompu par la pression arabo-islamique. Voir sur ce point les rappels de Salem Chaker, "Constantes et mutations dans l'affirmation identitaire berbère" (Revue de l'Occident Musulman et de la Méditerranée, 1987 : Berbères, une identité en construction).

${ }^{27} \mathrm{Ce}$ fut le cas pour certaines fractions kabyles.
} 
compte encore, non sans ambiguïté, dans la conscience collective des élites berbères) $)^{28}$.

Incontestablement c'est l'arabe qui pâtit, parce qu'il est la seule langue capable d'inférioriser le pôle roman. Si les autorités françaises ne cherchent pas à l'éradiquer (cela aurait constitué une fausse manœuvre), en revanche par un faisceau d'action très large (dont la recherche scientifique) elles mettent à nu les contradictions de ce pôle. De cette manière, la diglossie interne très forte de l'arabe (classique vs. dialectal), sans être "inventée" par la France est-elle particulièrement soulignée par ses chercheurs, ses enseignants, ses formateurs divers. Grande nouveauté, on s'intéresse de plus en plus à l'arabe dialectal, que l'on écrit et que l'on transcrit. On publie ainsi divers manuels et grammaires d'arabe dialectal. Cet intérêt apparemment bénin ou légitime dans l'esprit de la linguistique du temps est une véritable révolution culturelle dans la tradition des études arabes, et une bombe à retardement pour le pôle arabe ${ }^{29}$. L'arabe dialectal existera désormais et sortira des limbes où il était maintenu jusqu'alors. Autre nouveauté, la formation accrue d'arabisants européens qui, du point de vue colonial, a pour double finalité de contrecarrer le pouvoir des oulémas dans la sphère coranique et de préparer la rencontre culturelle historique entre arabe et français. Nous avons évoqué plus haut les modalités d'annexion de la Tunisie. On notera à ce propos que le premier Directeur de l'Enseignement public en Tunisie fut un arabisant français, M. Machuel "[.], professeur d'arabe à Oran, connu par ses travaux sur la langue arabe, et capable, par sa connaissance des moeurs, des coutumes et des idées musulmanes, de préparer le succès de notre domination. ${ }^{\prime 30}$.

\section{3-2-b. Interactions, identités et système tripolaire depuis les Indépendances.}

A partir des Indépendances le pôle arabe tente au fond de démonter le système hérité de l'Histoire profonde du Maghreb et charpenté par la colonisation; de reconquérir ainsi une partie du terrain, en affichant très souvent une volonté de reconquérir tout le terrain maghrébin. Il y parvient par certains aspects, mais en découvrant à plusieurs reprises ses propres limites.

Il faut dire que les directions pointées lourdement par la colonisation appellent régulièrement les excès inverses de ce pôle arabe auto-légitimé dans les années 60 à 80 . Mais ces excès sont bien sûr tout à fait exemplaires du système tripolaire. Par exemple la rejet en bloc d'une quelconque francité ou romanité, à plus forte raison d'une quelconque chrétienté du Maghreb. Car accepter ce type de

\footnotetext{
${ }^{28}$ Les études berbères initiées principalement en Algérie se sont étendues et enrichies au Maroc. C'est l'époque des travaux de Biarnay (Rif), Destaing (Sous), Laoust (Atlas), des "Archives berbères" publiées à l'initiative du Comité d'Etudes Berbères de Rabat, de la revue Hespéris qui perdra assez rapidement sa composante berbère après l'Indépendance du Maroc.

${ }^{29}$ On ne perdra pas de vue qu'à la fin du XIX ${ }^{\text {ème }}$ et le début du XX ${ }^{\text {ème }}$, les regards sur le Maghreb sont conditionnés par les mutations de la linguistique française : les études des dialectes et patois, de la tradition orale, ont pignon sur rue dans l'Université métropolitaine. La dialectologie est matière d'enseignement depuis les années 1880 et différents Atlas romans dont l'A.L.F. sont en cours de constitution. La découverte des parlers populaires d'Afrique du Nord (berbères et arabes) se trouve donc dans la prolongation de ce mouvement.

${ }^{30}$ L. Lanier, ouv. cité, p. 337.
} 
principe reviendrait dans bon nombre d'esprits à reconnaître l'existence même du pôle roman, alors que toutes les forces sont mises dans la balance pour faire du français une langue étrangère "comme les autres". L'arrière-plan politique tend bien sûr à radicaliser le plus souvent le discours anti-français et pro-arabe, car il s'agit alors de prendre le maximum de distances avec l'ancienne puissance coloniale. Sur le terrain statutaire et scolaire, le français est dans un premier temps poussé vers ce statut de langue étrangère que l'on veut lui donner sans l'abandonner pour autant. Sans grand succès, car cette attitude est ressentie par la plupart des acteurs du paysage linguistique comme caricaturale et non conforme aux faits. C'est ce que pensent beaucoup de gens dans les années 1970 à 80, mais une très forte auto-censure collective empêche généralement de le dire clairement (la décolonisation est encore trop proche), d'où des propos moyens ou relativement neutres tendant à cette époque à protéger l'arabe langue nationale sans trop agresser le français. Les adultes de cette époque, en effet, ont connu l'époque coloniale et la décolonisation, ce qui conditionne étroitement les propos tenus.

Depuis les années 80 une modification considérable des jugements semble encours. Elle est due à quelques facteurs combinés. Le premier est que les Etats indépendants réussissent mieux dans la francisation que la puissance coloniale. Avec la démocratisation de l'enseignement, le français touche plus profondément le Maghreb, élargissant ainsi sa base par rapport à la période coloniale. Il irradie désormais des classes sociales précédemment limitées à l'arabe dialectal et au berbère $^{31}$. D'autre part les classes d'âges atteintes sont de plus en plus décalées par rapport à la période coloniale. Le français est le plus souvent perçu comme une langue d'ouverture ${ }^{32}$ (cf. rôle 1), beaucoup moins comme la langue de l'oppression coloniale. D'autre part, comme on l'a déjà souligné, peut être constaté un début de dépôt régional du français, une voie d'appropriation maghrébine donc, proche mais à distinguer toutefois du français "pied-noir" ${ }^{33}$. Enfin, la position du français se renforce des échecs relatifs de l'arabisation. Celle-ci piétine dans les années 70 à 80 , passée l'euphorie de la décolonisation. Surtout, l'arabe a été potentiellement conçu comme une langue devant être à terme être étendue à l'ensemble de la région et à l'ensemble des rôles sans restriction, ce qui est contradictoire du système tripolaire. S'en suivent différentes phases de panne, de découragement, de retour en arrière ou de coups de force peu populaires, qui, en dépit des apparences, peuvent profiter aux deux autres pôles, français en premier lieu, berbère aussi. L'une des dernières manifestations spectaculaires de cette logique a été celle de l'interdiction législative de l'usage du français dans l'administration algérienne il y a quelques années, celle-ci semblant avoir renforcé en retour les soutiens mutuels du français et du berbère.

Deuxième rejet, connexe, celui du berbère. Vu depuis le pôle arabe, ce pôle, cette langue sont niés. Les plus ignorants peuvent percevoir le berbère comme une

\footnotetext{
${ }^{31}$ Phénomène remarqué par différents observateurs, dont Marc Gontard, La violence du texte, littérature marocaine d'expression française (Paris, L'Harmattan, 1981).

${ }^{32}$ Voir encore G. Grandguillaume, ouv. cité (cf. note 18).

${ }^{33}$ Pour le français "pied-noir" on dispose toujours notamment de André Lanly, Le français d'Afrique du Nord (Paris, Bordas, 1970).
} 
déformation inexplicable et inacceptable de l'arabe. De plus instruits introduiront le thème de la création ex-nihilo du problème berbère par la colonisation française, ce qui rejoint la jonction précédente français-berbère. Les deux sont alors renvoyés dos à dos, et pèse ainsi une suspicion chronique sur le berbère, régulièrement taxé de complicité. De plus souples ou cultivés traiteront enfin le berbère comme une langue régionale, une variété qui néanmoins ne saurait prétendre à une investiture statutaire dans le cadre d'un Etat moderne.

Dans ces conditions, sur le terrain statutaire et scolaire, pas de reconnaissance possible du pôle berbère, du moins jusqu'à très récemment. Aussi la jonction français-berbère a-t-elle été d'une certaine manière renforcée par les tendances totalitaires du pôle arabe. Pendant une vingtaine d'années (en gros de 1970 à 1990), par la force des choses, les départements de français sont devenus de véritables pépinières de travaux sur le berbère. Ainsi le pôle berbère se créait-il l'élite qui entend maintenant le réintroduire bien plus haut dans le système statutaire. De telle sorte qu'aujourd'hui le berbère apparaît comme un pôle fort et non plus comme un pôle statutairement dominé, ce qui était le cas durant les périodes précédentes. Pour reprendre notre classification en rôles, le pôle berbère tend aujourd'hui à asseoir son rôle 3 (reconnaissance statutaire de la fonction locale), à se donner le rôle 2 (reconnaissance statutaire de langue nationale), et, pourquoi pas, à empiéter sur le rôle 1 (véhicularité inter-maghrébine?).

\section{3-2-c. Éléments de prospective.}

Un tel système a peu de chances d'évoluer radicalement dans l'immédiat. Des indices de renouvellement possibles apparaissent pourtant.

Tout d'abord, l'histoire profonde des relations sociolinguistiques tend à authentifier la stabilité de la structuration tripolaire. C'est ce qu'on a tenté de montrer dans la première partie. Bien que les configurations changent, trois pôles perdurent depuis une vingtaine de siècles

Ensuite, en dépit des mouvements dans les répartitions statutaires observés depuis la colonisation française, le système semble à première vue bloqué. On le voit d'autant plus facilement que des actions conçues de manière radicale sur l'un des pôles (par exemple l'arabisation) ne provoquent pas de répercussions fondamentales sur l'ensemble. Il serait d'ailleurs plus juste de dire qu'un tel système est en situation d'équilibre, ou d'homéostasie, au moins au Maroc et en Algérie $^{34}$.

\section{Le cas tunisien.}

De ce point de vue, la Tunisie semble révéler ces dernières années un mouvement plus fin qui paraît en faire un front pionnier du paysage linguistique d'Afrique du Nord. Pour deux raisons semble-t-il complémentaires.

D'une part, le français s'y trouve dans une position solide, très implanté dans les circuits urbains et dans nombre de groupements sociaux. On pense tout d'abord

\footnotetext{
${ }^{34}$ Outre le recours à différents matériaux disponibles dans des publications récentes (en particulier sur l'Algérie), je m'appuie dans cette partie sur des enquêtes épilinguistiques menées par mes étudiants à l'E.N.S. de Meknès (1984-1987) ou à l'E.N.S. de Sousse (1987-1991), ainsi que sur quelques enquêtes personnelles dans ces deux pays et durant la même période.
} 
à la bourgeoisie tunisienne, notoirement et profondément francophone, qui lit, pense, éduque ses jeunes en français, assumant généralement bien cette composante sociolinguistique de son identité. Mais d'autres groupements sociaux sont touchés par des agences sociolinguistiques convergentes telles que l'Ecole ou le Tourisme. Dans le tourisme, beaucoup de tunisiens travaillent en effet directement en français, et -signe des temps- dans différentes langues germaniques d'Europe du Nord (voir plus loin les tendances concomitantes au glissement vers l'anglophonie). Ajoutons enfin la diffusion directe de plus en plus importante de programmes télévisuels français depuis les années 80 , dont la diffusion en quasi-totalité de la chaîne publique française Antenne 2, devenue France $2^{35}$.

D'autre part, le pôle berbère est en extinction. Le contraste est saisissant avec l'Algérie ou le Maroc. Pour le tunisien moyen le berbère n'existe pas en tant que langue: on se pose peu de questions de ce point de vue et l'on fait généralement comme si la langue était déjà morte. En masse, le berbère est au mieux parlé par 1 à $2 \%$ de la population (plus ou moins couramment). C'est en outre, comme toutes les langues moribondes, une langue cachée, de l'intimité voire de la honte, dominée sur la place publique par l'arabe local. Cette langue, là où elle est encore un peu parlée est désormais fragmentée puisque les points berbérophones ou réputés tels sont disséminés (sud tunisien et Ile de Djerba). Ce défaut de continuité est prolongé par une absence à peu près totale de conscience ethnolinguistique collective berbère. Les derniers berbérophones vivent très calmement la disparition prévisible de leur idiome ancestral.

Dans la pratique, il ne reste donc désormais en Tunisie que deux pôles face à face: arabe et français. Le principe d'alliance plus haut invoqué n'est alors plus possible, par défaillance du berbère. S'en suit alors une réaccommodation en chaîne du système.

Le pôle arabe récupère logiquement une série de fonctions du berbère. Les informateurs tunisiens, derrière des propos relativement calmes sur la langue, révèlent une très forte tendance à faire de l'arabe la langue de l'identité culturelle et religieuse, une sorte de réservoir permanent de riposte identitaire à toute acculturation occidentale trop violente ou rapide (cumul rôle $3+$ rôle 2 ). Ceci permet à de nombreux informateurs de porter en même temps un constat de relative déficience de l'arabe (thèmes fréquents de l'échec de l'arabisation, de l'inaptitude à l'universalité moderne etc.) tout en s'y référant régulièrement comme garantie d'authenticité nationale et culturelle.

On assigne ouvertement le rôle 1 au français. D'après les informateurs, c'est principalement cette langue qui permet à la Tunisie de maintenir sa position internationale politiquement, économiquement et symboliquement. Pourtant ce rôle est assez souvent contesté par l'anglais, non dans la réalité des faits mais dans les projections sociolinguistiques effectuées par une portion des tunisiens cultivés.

\footnotetext{
${ }^{35} \mathrm{~A}$ la fin des années 80, le dossier télévisuel était considéré comme un dossier prioritaire par l'Ambassade de France. On soulignera que la chaîne française était déjà largement relayée par la deuxième chaîne tunisienne (RTT), mais la diffusion directe semble avoir rencontré un profond succès, faisant en particulier chuter assez brutalement l'audience de la télévision italienne (la RAI Uno, reçue directement dans le nord de la Tunisie depuis plusieurs années).
} 
A terme (mais sans pouvoir préciser les délais et mécanismes), certains verraient bien l'anglais remplacer le français comme langue d'ouverture. Ce raisonnement mérite que l'on s'y arrête. Il constitue la variante négative d'une ligne de faille plus générale dans la francophonie. Ici comme ailleurs on observe (à tort ou à raison) que l'anglais est en passe de supplanter le français comme langue véhiculaire à l'échelle planétaire. Mais alors qu'ailleurs on s'inquiète plutôt de l'éventualité de devoir devenir un jour anglophone "forcé", l'idée (pour l'instant très minoritaire) est que l'on pourrait shunter en allant directement à l'anglais. Cette stratégie serait apparemment utilitaire. A y regarder de plus près, elle pourrait correspondre à un moyen détourné de se débarrasser du français, au moment où cette langue tend à être intégrée comme partenaire "normal" du paysage tunisien et dans la mesure où l'arabe ne semble pas en mesure d'arriver à cette évacuation par ses moyens propres.

On touche donc à une question brûlante, celle de l'identité tunisienne. Tout semble se passer comme si un constat de blocage arabe vs. français sans solution immédiate était identifié par le plus grand nombre. L'absence du berbère comme troisième pôle radicalise l'affrontement, ce qui dans les propos conduit les informateurs à cultiver le paradoxe. On reconnaît ainsi le français comme langue presque "maternelle" des tunisiens, pour le rejeter quelques minutes plus tard comme expression non convenable de l'identité profonde. Parallèlement, on assigne cette fonction d'identification culturelle à l'arabe tout en l'évacuant sur le plan des usages économiquement porteurs.

La relative réussite du français correspond à une descente du pôle vers le rôle 2. On a là l'illustration d'un processus d'appropriation de cette langue qui a comme corollaire l'apparition possible d'une autre langue dans le rôle 1 . Cette langue "à venir" ne pouvant être semble-t-il l'arabe, l'éventualité d'une installation de l'anglais dans le paysage linguistique présenterait alors divers avantages. Celui d'intégrer directement la Tunisie aux réseaux présumés du pouvoir économique et culturel mondial, l'anglais étant vu comme langue de promotion économique planétaire. Celui surtout d'avoir dans le rôle 1 une langue non maghrébine, vraiment étrangère et à la limite choisie, surtout reçue comme distante de la personnalité maghrébine collective. Car le français n'est plus perçu comme une langue étrangère par la majorité des tunisiens (et maghrébins) cultivés. Nombreux sont ceux qui le disent "naturel", "inné", "presque maternel". Il y a dans tous les cas, à l'évidence, une relation affective au français, pas seulement une relation de nécessité. On pourrait dire en un mot que le point faible du français aujourd'hui est son relatif succès et ses progrès quarante ans après l'indépendance du pays.

\section{Les cas algérien et marocain.}

Ils représentent toujours la mécanique tripolaire "classique", à deux degrés différents de radicalisation. Ce qui distingue nettement ces pays de la Tunisie. Ici le conflit arabe/français est devenu indissociable d'un second conflit berbère/arabe qui est passé récemment au premier plan. Bien entendu, ce conflit n'est pas nouveau, mais on va de plus en plus nettement vers un affrontement violent qui 
déborde du cadre de la revendication linguistique et culturelle de la communauté kabyle, puisqu'il s'agit d'elle en particulier ${ }^{36}$.

En fait, depuis la fin des années 70 comme on l'a vu plus haut, l'arabe est contesté par les deux autres pôles. Et de principalement passifs les conflits sont devenus aujourd'hui actifs.

Etait passif le pôle français tant que l'arabe politiquement dominant tendait à le repousser sur les terrains statutaires, ce qui correspond en gros à une période nationaliste et panarabe, en Algérie et ailleurs. Les années 80 révélant des échecs relatifs et divers reculs des politiques globales d'arabisation, en contrecoup la domination politique arabo-islamique sur le pôle berbère a été de plus en mal vécue par les berbérophones, d'autant que cette domination se traduisait par une répression claire de tout ce qui semblait pouvoir déboucher sur un séparatisme berbère. Parallèlement, l'évacuation largement dogmatique du français devenait de moins en moins justifiable. Très spontanément, l'affinité des deux pôles français et berbère s'est rematérialisée, après une phase d'éloignement d'une trentaine d'années $^{37}$. Mais dans la plupart des cas, on s'attache à évacuer désormais tout signe d'allégeance, en évitant généralement de camper une quelconque collusion berbéro-française. Il est vrai que la lutte berbère passe en grande partie par le français (voir 3-2-b), que les deux langues sont également l'objet de restrictions multiples par l'arabe, mais on reste discret sur les jonctions manifestes ou potentielles de ce couple de dominés. Il n'en reste pas moins que partisans de la berbérophonie et de la francophonie sont descendus dans la rue dans les années 90, ensemble, pour la bonne raison qu'il s'agissait en grande partie des mêmes ${ }^{38}$.

On retrouve dans cette liaison de facto quelque chose d'ancien, c'est le principe même de coalition face au pôle politiquement dominant. En revanche un changement s'est produit, qui explique en grande partie la radicalisation actuelle. Il s'agit en fait d'une mutation au sein du pôle arabe, qui rejaillit sur le reste du système et que l'on doit à nouveau faire remonter aux périodes antérieures. L'ascension de l'arabe jusqu'aux années 70 se fait généralement sans rapport direct avec le noyau cultuel islamique auquel cette langue est originellement attachée,

\footnotetext{
${ }^{36}$ Voir à nouveau Salem Chaker, "Langue et culture berbères en Algérie depuis 1988" (Cahiers de linguistique sociale, 1993 : Minoration au Maghreb).

${ }^{37}$ On doit souligner que cette phase d'éloignement entre pôle berbère et pôle français, accompagnée d'un rapprochement de fait entre pôle arabe et pôle berbère, correspond à la place prépondérante occupée par la Kabylie dans la lutte anti-coloniale. Néanmoins, à l'intérieur du mouvement de libération nationale les clivages politiques semblent avoir largement recoupé le clivage ethnique Arabes vs. Berbères. Voir encore Salem Chaker, "Constantes et mutations dans l'affirmation identitaire berbère", article cité (note 26).

${ }^{38} \mathrm{~A}$ une époque où M. Aït Ahmed (Front des Forces Socialistes) faisait ses discours en français, une manifestation organisée par le même F.F.S. mit dans les rues d'Alger, le 27 décembre 1990, un demi-million de personnes environ. L'Assemblée populaire algérienne venait en effet d'adopter un texte portant généralisation de l'emploi de l'arabe dans l'ensemble des administrations publiques. L'acte visait donc prioritairement le français, d'usage très courant en particulier dans la haute administration. Pourtant, pour la plupart des observateurs fut évidente la jonction berbère-français dans un cadre global de mise en échec de "la montée de l'obscurantisme". Les slogans les plus fréquents (contre "l'arabisation au rabais" et "l'intolérance politique et religieuse", pour "le tamazight à l'école", "ne touchez pas à nos langues" etc.) résument on ne peut mieux la tension de la mécanique tripolaire en Algérie. Voir également la note 19 pour le Maroc.
} 
bien que les constitutions précisent que l'arabe est seule langue, et l'islam seule langue des Etats maghrébins. Il y a même place, on le sait, pour un arabisme laïc, progressiste, matérialiste parfois; les politiques scolaires maghrébines distinguent alors enseignement originel et enseignement moderne bilingue. C'est en effet la période durant laquelle l'arabe doit fonder des Etats modernes et affranchir ceuxci de la domination occidentale. Le paradoxe relatif est que l'arabe tend pour cette raison à investir des registres occupés jusqu'alors par le français, sans succès total. Dans les années 80 on s'aperçoit aussi que la dépendance des Etats maghrébins par rapport à l'Europe s'accroît (dépendance économique, politique et culturelle), une Europe elle-même entrée dans une spirale d'unification. La France et le français restent l'intermédiaire, la passerelle prioritaire vers cette intégration mondiale, en dépit des avancées techniques de l'anglais (partout au Maghreb comme dans la francophonie). Ceci renforce donc la place du français comme langue d'ouverture économique et provoque une inversion radicale dans l'organisation du pôle arabe. La langue arabe n'ayant pas garanti par sa propre dynamique l'indépendance du Maghreb, se met en place alors un raisonnement qui remet à l'ordre du jour la riposte identitaire en replaçant l'arabe dans le cadre préalable du raisonnement islamique. Ce raisonnement, qui se précise sous nos yeux, revigore le pôle mais en même temps pourrait condamner l'arabe à refluer de rôles patiemment investis durant les décennies précédentes. Car la langue arabe n'est plus cette fois conçue comme le moteur du pôle, elle en serait plutôt l'outil, forcément secondarisée par rapport à la dynamique d'identification sociale cultuelle. La logique interne du pôle arabe serait donc plutôt en ce moment une logique de repli et d'enfermement.

C'est pour finir cette logique d'inversion islamo-arabe qui conduit désormais à de véritables affrontements vécus comme des choix de société. Militer ouvertement pour le berbère (chose pratiquement impossible dans les années 70), militer pour la survie statutaire du français, sont désormais des actes d'engagement par rapport aux scénarios d'évolution à court et moyen-terme de la société algérienne. Tous ceux qui réclament reconnaissance et droits effectifs pour les pratiques en berbère ou en français développent en général le sentiment net de mener ce faisant un combat historique contre l'obscurantisme et pour la tolérance. Comme on le sait, ces comportements sont bien plus visibles et tendus en Algérie; Mais au Maroc, une logique tout à fait comparable se développe ${ }^{39}$.

Dès lors qu'on interfère directement avec la politique et ses ressorts intimes, il est difficile de prévoir des évolutions trop précises de ce système tripolaire. L'ordre politique et l'ordre sociolinguistique, tout en échangeant activement, disposent de modalités d'enchaînement et de vitesses de réaction différentes. On peut toutefois ébaucher quelques pistes d'évolution du système sociolinguistique considéré.

Le scénario le plus vraisemblable reste le maintien du système tripolaire, mais peu probablement sous ses formes actuelles. La position du berbère devrait principalement se consolider dans l'avenir. Il y a en effet en Algérie comme au Maroc une remontée profonde du pôle, qui n'a rien à voir avec une revendication

${ }^{39}$ Voir notes 38 et 19. 
seulement régionaliste ou folklorique. Cette lame de fond vient de beaucoup plus loin et arrive beaucoup plus fort, encadrée par des élites formées. On peut donc présumer que les berbérophones d'Algérie mais aussi du Maroc s'accommoderont très mal de quelques strapontins. Tout se passe en effet comme si la très longue impasse du "Maghreb Arabe" sur ses larges majorités ou minorités berbères ${ }^{40}$ conduisait au résultat contraire: le berbère tend à revendiquer désormais un statut de langue nationale (rôle 2).

Le deuxième scénario est celui de la prise de pouvoir islamiste, qu'elle soit fragmentaire ou totale, progressive ou très rapide. Scénario que nombre d'observateurs politiques voient planer sur l'Algérie en priorité, et qu'il faut évoquer d'un point de vue prospectif. Dans cette hypothèse, la question des choix sociolinguistiques deviendrait brûlante. En dépit de leurs orientations politiques diverses et des nombreux traits différentiels qu'ils se reconnaissent, les Etats et les peuples actuels du Maghreb ont en commun de se trouver dans une situation de continuité par rapport à l'Histoire profonde, par rapport à la colonisation puis à l'attraction européenne. Les formes actuelles du système tripolaire en sont l'expression. Une réorientation politique fondamentaliste devrait logiquement marquer une rupture hautement symbolique vers des choix unanimistes musulmans et pro-arabes. En matière linguistique, ceux-ci pourraient être spectaculaires (prise de décrets sur la langue) ou se traduire par une politique d'interdiction au moins "en surface", faute de pouvoir agir directement sur la base ethnique, sociale et économique des langues en premier visées: français et berbère. Dans le cas du français il faudrait alors faire disparaître ou au moins reculer significativement les signes visibles de la francophonie (affichages, publications, presse, bilinguismes d'Etat etc. ${ }^{41}$. Dans le cas du berbère, tous les ingrédients de l'affrontement sembleraient réunis en Algérie, car les logiques respectives du pôle arabe et du pôle berbère seraient en totale contradiction cette fois. On a évidemment le plus grand mal à imaginer un pouvoir intégriste tolérer l'expression directe de l'identité berbère, même sous des formes atténuées.

Reste la question scolaire. Un pouvoir de ce type peut-il du jour au lendemain évacuer la fausse "langue étrangère" que constitue le français? La réponse est probablement non, du moins dans l'immédiat. D'une certaine manière, il faudrait encore assurer un minimum de continuité en attendant la solution de remplacement viable, ou faute de cela s'engager dans un effondrement économique qui se retournerait probablement contre le pouvoir qui déciderait ainsi. On retrouve alors l'hypothèse moyenne, celle du renforcement voire de l'exclusivité à terme de l'anglais dans le système scolaire. D'une part, comme on l'a souligné, l'aspiration à l'anglais "langue internationale" monte régulièrement à travers l'ensemble du Maghreb depuis la fin des années 70. Quand le système tripolaire boîte (cas de la Tunisie), ces potentialités de remplacement s'accentuent.

\footnotetext{
${ }^{40}$ On estime généralement qu'environ $1 / 3$ des algériens (soit 9 millions de personnes) sont actuellement berbérophones. Au Maroc, c'est vraisemblablement la moitié au moins de la population (soit plus de 10 millions) qui le parle. Mais on dispose en réalité de très peu d'éléments sûrs, tant pour l'Algérie que pour le Maroc.

${ }^{41}$ De ce point de vue, on interprète souvent la loi sur l'emploi de l'arabe en Algérie (1990) comme une concession anticipée à l'électorat intégriste (voir note 38 ).
} 
Le remplacement plus ou moins rapide du français par l'anglais présenterait ainsi deux avantages : celui de démontrer une rupture par rapport au monde occidental le plus proche (la France dans l'Europe) et celui de maintenir sans trop d'affectivité un fill linguistique économiquement porteur ${ }^{42}$.

Autre question, et non des moindres, celle de l'organisation statutaire au sein du pôle arabe. Celui-ci, dans une logique d'autosuffisance, n'aurait d'autre solution que de réduire la fracture diglossique de l'arabe. Ce qui veut dire qu'un pouvoir de ce type devrait donner aux arabes dialectaux les références statutaires qui leur ont toujours été refusées dans l'Histoire de l'arabisme au Maghreb. Il y a certainement là une contradiction majeure de principe, l'arabe dialectal comme le berbère relevant automatiquement des parlures triviales et impures. Mais en revanche, le recrutement très populaire des mouvements politiques considérés, pour des raisons statistiques et matérielles, pourrait après tout déboucher sur certaines formes de reconnaissance du dialectal dans les circuits de l'Etat. Ces deux schémas peuvent être combinés, mais des deux devrait dominer et perdurer celui de l'exclusion du dialectal. L'autre schéma (tolérance et intégration) suppose une véritable révolution culturelle difficile aujourd'hui et loin d'être évidente dans un proche avenir ${ }^{43}$.

\section{Conclusion: de l'insécurité du Maghreb.}

Le modèle d'observation diachronique que nous proposons tend à fournir un cadre général d'interprétation des mouvements sociolinguistiques. Qu'il s'agisse des "allers-retours" de l'arabisation, de l'insécurité identitaire vis à vis du français ou du mouvement de panique face au regain berbère, les gens qui parlent de la langue au Maghreb soulignent très régulièrement les apparentes "absurdités" du système. Car vus de trop près, plus exactement de l'intérieur, les mouvements sociolinguistiques paraissent avant tout incohérents, en rupture fréquente tant avec la logique de l'Etat qu'avec les fibres identitaires de ceux qui parlent.

Avec un contrôle en profondeur, les phénomènes paraissent bien plus cohérents. En 1972, William Labov avait trouvé une formule très appropriée dans son titre "On the use of present to explain the past", car les mécanismes du changement linguistique à travers nous et sous nos yeux reconduisent des principes habituellement rejetés dans la diachronie profonde ${ }^{44}$. La perspective inverse est nécessaire. C'est celle que nous choisissons ici : l'observation des fonctionnements du système à travers le temps permet de mieux comprendre ce qui se passe sous nos yeux, et ce qui pourrait se passer dans l'avenir.

\footnotetext{
${ }^{42}$ On ajoutera que de nombreux cadres, scientifiques ou politiques (ceux des mouvements intégristes en particulier) séjournent et se forment en domaine anglo-saxon (Etats-Unis et GrandeBretagne tout particulièrement). Leur regard sur les langues internationales au Maghreb en est nécessairement modifié.

${ }^{43}$ Mohamed Benrabah après d'autres (L'arabe algérien véhicule de la modernité, Cahiers de linguistique sociale, 1993, ouv. cité) observe justement un principe de dévalorisation de la seule vraie langue maternelle des arabophones d'Algérie, qui engendre une véritable "haine de soi".

${ }^{44}$ William Labov, "On the use of present to explain the past", $\mathrm{XI}^{\text {ème }}$ congrès des linguistes. Bologne, 1972.
} 
$\mathrm{Au}$ terme de ce parcours, retenons quelques points clefs. Si le paysage linguistique maghrébin semble bien fonctionner sur la base d'un schéma tripolaire trans-chronique, il est important de souligner que ce schéma conduit à mettre en présence des pôles ou langues toujours en situation d'insécurité. Mais cette insécurité ne condamne pas le pôle en question, plus exactement elle en relativise le poids et en limite constamment la portée. La force d'un tel système est qu'il semble en général pouvoir éviter les situations d'affrontement dialectique typiques de systèmes principalement bipolaires comme ceux de la France ou de l'Espagne, dans le voisinage immédiat du Maghreb. Dans ces deux cas bien connus au sein de la Romania, une langue expression de l'Etat avance lourdement et tend à régler sur la base de sa propre logique les fonctionnements du paysage linguistique. Illustre ce schéma la situation diglossique des régions méridionales de la France étudiée par Robert Lafont et d'autres il y a quelques années, où deux langues principales et leurs variétés sont en présence ${ }^{45}$. Dans le cas du Maghreb, on aura compris que le système implique à tout moment un dédoublement des cibles. Chacune des trois langues est limitée tôt ou tard par les deux autres, et, d'autre part, la concurrence dans chacun des pôles peut être rude (français vs. espagnolitalien, ou arabe classique vs. arabe dialectal). Par une sorte de paradoxe on peut dire que le trilinguisme maghrébin ferme et stabilise le paysage linguistique en émoussant les forces de renouvellement. Est-ce une force ou une faiblesse? L'avenir et les Maghrébins eux-mêmes donneront la réponse. Mentionnons seulement deux attitudes très courantes et opposées sur ce point.

Faiblesse : en tolérant et en pratiquant plusieurs langues, les Maghrébins en fait n'en parlent aucune correctement; la situation de plurilinguisme les appauvrirait donc.

Force : le $\mathrm{XXI}^{\text {ème }}$ siècle exigera des locuteurs qu'ils pratiquent plusieurs langues dans leurs activités quotidiennes et au cours de leur vie. De ce point de vue les Maghrébins trouveront leur compte pour avoir intégré les préalables mentaux du multilinguisme.

L'insécurité des pôles comporte un miroir identitaire. Les personnalités collectives apparaissent en effet comme partagées, parfois déchirées. De nos jours, la première grande faille visible est celle qui passe entre l'européanité et la maghrébinité. Elle traverse nombre de propos et la plupart des maghrébins un tant soit peu cultivés se voient à cheval sur les deux principes d'aspiration culturelle. On reconnaît le déjà vieux débat sur l'acculturation française du Maghreb. La maghrébinité est également partagée. Elle prend sa source dans l'arabité locale ou dans la berbérité, elle est donc par définition polémique. Le couple arabo-berbère a toujours été un couple tendu, fondé sur des extrêmes berbérophones ou arabophones qui s'ignorent ou se rejettent, également fondé sur des personnalités en forte crise identitaire chez les nombreux diglottes en cours d'arabisation. La maghrébinité exclut d'autre part une participation trop nette à l'arabité globale : nombre de propos montrent en effet une forte méfiance par rapport aux références identitaires arabes orientales. Est clairement visé le Machrek (monde arabe

\footnotetext{
${ }^{45}$ Pour ce type de situation, cf. la revue Langages, $\mathrm{n}^{\circ} 61$ : Bilinguisme et diglossie (dirigé par J.-B. Marcellesi, 1981), en particulier Ph. Gardy \& R. Lafont, "La diglossie comme conflit, l'exemple occitan".
} 
oriental à partir de l'Egypte), vu par beaucoup comme un repoussoir identitaire dans lequel les Maghrébins ne sauraient se reconnaître. Ce type de position se rencontre très fréquemment chez des arabophones purs, ce qui mérite d'être souligné. Mais le rejet n'est jamais intégral, comme vis à vis de l'Europe : l'attraction et la répulsion vont de concert et tendent également à la neutralisation, comme les dynamiques des trois pôles

$\mathrm{Au}$ sein et autour des pôles identitaires, les fractures sont également nettes. A une certaine époque, le français pouvait être vu comme une langue de collaboration des élites maghrébines. Il discriminait alors les élites et le pouvoir du peuple ${ }^{46}$. Un type de difficulté comparable touche désormais l'arabe et la culture arabe et certains, arabophones en tête y voient une nouvelle forme de domination ou d'acculturation. Le clivage ancien classique vs. dialectal, au lieu de se combler semble au contraire s'être aggravé. Pas tellement dans les faits car la scolarisation, comme dans le cas du français, a élargi la masse relative des locuteurs capables de s'exprimer en arabe soutenu. Par contre, dans leur grande majorité, les acteurs disent avoir des difficultés à se reconnaître dans une langue et une culture qui leur restent passablement étrangères, auxquelles ils ont le sentiment très net d'accéder par une sorte d'exercice de style. Près de 40 ans plus tard, le propos de l'écrivain tunisien Béchir Khraief reste en gros valable : "d'une part, chaque arabe emploie l'arabe dialectal dans sa vie quotidienne, et, d'autre part quand il désire écrire, il s'engage dans un continent étranger [.]"47.

Dernier pan, la culture et l'identité berbères. Partout où le berbère est réellement parlé (Maroc, Algérie) s'affirment des canaux d'auto-reconnaissance et de revendication. Etre Berbère, c'est se voir différents des autres, par la langue bien entendu, mais au-delà par les schémas d'organisation sociale que véhicule la langue, la tradition orale, la structure de la famille ou de la tribu, les manières de se nourrir ou de se comporter. Bref, c'est sur la totalité du tissu identitaire que se fondent désormais les jeunes élites berbères, des élites de plus en plus souvent formées dans l'Enseignement supérieur marocain ou algérien ${ }^{48}$.

Où se trouve donc l'identité maghrébine? En stricte objectivité elle apparaît fragmentée dans la continuité des trois constituants fondamentaux ou pôles. Sur le terrain des réalités langagières et identitaires, chacun des pôles, avec des taux différents suivant les périodes, a visiblement fondé une personnalité collective à facettes. Cette intégration pluri-culturelle d'ordre ethnolinguistique est en décalage avec les logiques culturelles planétaires, à propension unanimistes, qu'il s'agisse de la logique euro-américaine ou de la logique arabo-islamique. Attaquer trop nettement l'une de ces trois composantes voue à l'échec. Tout se passe en fait comme si le Maghreb montrait au reste du monde, tantôt calmement, tantôt dans

\footnotetext{
${ }^{46} \mathrm{C}^{\prime}$ est là un thème constant de la littérature de l'acculturation algérienne dans les années 60 et 70. Voir par exemple Abdelmajid Meziane, Les élites castes (Révolution africaine, 1969), cité par Jean Déjeux, Culture algérienne dans les textes (O.P.U.-Publisud, 1982), une anthologie très représentative qu'il faut consulter.

${ }^{47}$ Propos de 1959, cité par Zohra Riahi, Emploi de l'arabe et du français par les élèves du secondaire (Cahiers du CERES -Tunis, 1970), repris dans Language in Tunisia, ouv. cité.

${ }^{48}$ Il est psychologiquement très important pour elles que les élites berbéristes soient en bonne partie formées et en fonction sur place, ce qui les met en position d'irriguer directement et légitimement les terrains idéologiques marocain et algérien.
} 
la violence, le plus souvent avec difficulté, qu'une vraie culture de synthèse est toujours possible.

A la condition d'étendre le raisonnement au berbère, on laissera conclure Salah Garmadi, linguiste tunisien trop tôt disparu : "Il faut donc prendre conscience, se libérer et se sentir tout entier. Si nous sommes des bâtards littéraires, il faut que nous assumions cette bâtardise comme une des caractéristiques essentielles de notre être et que nous en soyons fier, en attendant, dans la lutte quotidienne, de nous réapproprier une identité nationale positive et unitaire dynamique. ${ }^{49}$.

${ }^{49}$ Propos tenu dans la revue Alif, 1971. De Salah Garmadi, voir plusieurs contributions dans les Cahiers du CERES (Tunis). 